\title{
Effect of Planting Dates and Cultivars on Floral Characters of Gladiolus (Gladiolus grandiflorus) under Chhattisgarh Plains
}

\author{
T. Tirkey ${ }^{1}$, Sameer Tamrakar ${ }^{2}$, Gaurav Sharma $^{1}$ and Mukesh Sahu ${ }^{1 *}$ \\ ${ }^{1}$ Department of Floriculture and Land Scape Architecture, CoA, IGKV, Raipur (492012) India \\ ${ }^{2}$ Dau Kalyan Singh College of Agriculture and Research Station, Bhatpara, IGKV, \\ Raipur (493118) India \\ *Corresponding author
}

\begin{tabular}{|l|}
\hline K e y w o r d s \\
Gladiolus, Planting \\
Dates, Genotypes \\
\hline Article Info \\
\hline $\begin{array}{l}\text { Accepted: } \\
\text { 18 May } 2018 \\
\text { Available Online: } \\
\text { 10 June } 2018\end{array}$ \\
\hline
\end{tabular}

\section{A B S T R A C T}

The present investigation entitled Effect of planting dates and cultivars on floral characters of gladiolus (Gladiolus grandiflorus) under Chhattisgarh plains was Horticulture Farm, Department of Horticulture, College of Agriculture, Indira Gandhi Krishi Vishwavidyalaya, Raipur (C.G.) India during Rabi season 2011-12 and 2012-13. Having four different planting times $\left(30^{\text {th }}\right.$ September, $15^{\text {th }}$ October, $30^{\text {th }}$ October, $15^{\text {th }}$ November $)$ and six gladiolus genotypes (American Beauty, White Prosperity, Candyman, Dull Queen, Her Majesty, and Red Majesty). The field experiments were laid out in Factorial Randomized Block Design with three replications having 24 treatment combinations. The treatment $30^{\text {th }}$ September with cultivar Dull Queen $\left(\mathrm{D}_{1} \mathrm{~V}_{4}\right)$ was showed minimum days (52.50) required for spike emergence, the longest spike $(88.47 \mathrm{~cm})$ was recorded with the interaction of $30^{\text {th }}$ September planting with cultivar Candyman $\left(D_{1} V_{3}\right)$, the longest rachis $(67.43 \mathrm{~cm})$ was obtained in planting on $15^{\text {th }}$ October with cultivar Candyman $\left(\mathrm{D}_{2} \mathrm{~V}_{3}\right)$. Planting of $30^{\text {th }}$ September with American Beauty $\left(\mathrm{D}_{1} \mathrm{~V}_{1}\right)$, produced greater number of spike per plant (2.77), earlier floret opening (62.50) was recorded with the interaction of $30^{\text {th }}$ September planting with cultivar Dull Queen $\left(\mathrm{D}_{1} \mathrm{~V}_{4}\right)$, maximum number of floret per spike (15.50) was recorded with the interaction of planting on $15^{\text {th }}$ October with cultivar Candyman $\left(\mathrm{D}_{2} \mathrm{~V}_{3}\right)$, maximum flower diameter (11.70) was recorded with the interaction of $30^{\text {th }}$ September with cultivar Red Majesty $\left(D_{1} V_{6}\right)$, and the longest internodal length $(5.00$ $\mathrm{cm}$ ) was noted under interaction of planting on $30^{\text {th }}$ September with cultivar Candyman $\left(\mathrm{D}_{1} \mathrm{~V}_{3}\right)$.

\section{Introduction}

The genus Gladiolus L. belongs to the family Iridaceae and is a native of South Africa and Asia Minor. There are more than 180 known species of the gladiolus today, but only few of them are found in most of the gardens. Gladiolus is a flower of glamour and perfection which is known as the queen of bulbous flowers due to its flower spikes with florets of massive form, brilliant colours, attractive shapes, varying size and excellent shelf life. It is one of the most important bulbous ornamental, occupying fifth positions in International Floriculture Trades (Sharma and Sharga, 1994). Gladiolus is grown as 
flower bed in garden and used in floral arrangements for interior decoration as well as making high quality bouquets (Lepcha et al., 2007).

Gladiolus is a very popular bulbous flowering plant and it has a long and noble history. The name gladiolus was derived from the Latin word "gladiolus" means sword and hence it is often called as "sword lily" owing to the shape of its leaves. Gladiolus is very rich in its varietal wealth and every year there is an addition of new cultivars. Though many genotypes of gladiolus can be grown in particular agro-climatic region, all are not suited for cut flower purpose or for garden display or for exhibition purposes thus, there is a need for evaluation of genotypes and suitable time of planting for particular agroclimatic condition. Several genotypes of gladiolus, in Chhattisgarh, need to investigate to standardize a suitable planting date for attaining an optimum pre flowering growth which is a pre requisite for the production of standard cut flower and corms. Gladiolus is most interesting flower one could choose to grow due to its florets of brilliant colours, attractive shapes and varying sizes suited to different purposes. Successful production of quality flower depends much upon the planting time which indirectly influences the keeping quality (Usha Bala, 2002).

\section{Materials and Methods}

The present investigation was conducted in Horticulture Farm, Department of Horticulture, College of Agriculture, Indira Gandhi Krishi Vishwavidyalaya, Raipur (C.G.) India during Rabi season 2011-12 and 2012-13. Healthy and uniform size corms of $4-5 \mathrm{~cm}$ diameter were planted at different planting dates. The field experiments were laid out in Factorial Randomized Block Design with three replications having 24 treatment combinations $\left(\mathrm{D}_{1} \mathrm{~V}_{1}, \mathrm{D}_{1} \mathrm{~V}_{2}, \mathrm{D}_{1} \mathrm{~V}_{3}\right.$,
$\mathrm{D}_{1} \mathrm{~V}_{4}, \mathrm{D}_{1} \mathrm{~V}_{5}, \mathrm{D}_{1} \mathrm{~V}_{6}, \mathrm{D}_{2} \mathrm{~V}_{1}, \mathrm{D}_{2} \mathrm{~V}_{2}, \mathrm{D}_{2} \mathrm{~V}_{3}, \mathrm{D}_{2} \mathrm{~V}_{4}$, $\mathrm{D}_{2} \mathrm{~V}_{5}, \mathrm{D}_{2} \mathrm{~V}_{6}, \mathrm{D}_{3} \mathrm{~V}_{1}, \mathrm{D}_{3} \mathrm{~V}_{2}, \mathrm{D}_{3} \mathrm{~V}_{3} \mathrm{D}_{3} \mathrm{~V}_{4}, \mathrm{D}_{3} \mathrm{~V}_{5}$, $\mathrm{D}_{3} \mathrm{~V}_{6}, \mathrm{D}_{4} \mathrm{~V}_{1}, \mathrm{D}_{4} \mathrm{~V}_{2}, \mathrm{D}_{4} \mathrm{~V}_{3}, \mathrm{D}_{4} \mathrm{~V}_{4}, \mathrm{D}_{4} \mathrm{~V}_{5}, \mathrm{D}_{4} \mathrm{~V}_{6}$ ) comprising of four different planting times i.e. $30^{\text {th }}$ September $\left(D_{1}\right), 15^{\text {th }}$ October $\left(D_{2}\right), 30^{\text {th }}$ October $\left(D_{3}\right), 15^{\text {th }}$ November $\left(D_{4}\right)$ And six gladiolus genotypes viz. American Beauty $\left(\mathrm{V}_{1}\right)$, White Prosperity $\left(\mathrm{V}_{2}\right)$, Candyman $\left(\mathrm{V}_{3}\right)$, Dull Queen $\left(\mathrm{V}_{4}\right)$, Her Majesty $\left(\mathrm{V}_{5}\right)$ and Red Majesty $\left(\mathrm{V}_{6}\right)$. Observations were recorded on Flower yield and quality parameters viz. Days to spike emergence, Length of spike $(\mathrm{cm})$, Rachis length $(\mathrm{cm})$, Number of spike per plant, Days to first floret open, Number of floret per spike, Diameter of floret $(\mathrm{cm})$ and Internodal length.

\section{Results and Discussion}

\section{Flower yield and quality parameters}

\section{Days to spike emergence}

Dates of planting showed significant influence on days to spike emergence during both the years as well as pooled mean basis. The earlier spike emergence (58.38, 59.94 and 59.16 days) was observed in planting on $30^{\text {th }}$ September $\left(D_{1}\right)$, The maximum days $(60.33$, 62.55 and 61.45 days) required to spike emergence were observed in planting on $15^{\text {th }}$ November $\left(\mathrm{D}_{4}\right)$. Amongst the different cultivars, significantly the minimum days (52.25, 54.58 and 53.42) were taken by cultivar Dull Queen $\left(\mathrm{V}_{4}\right)$ to spike emergence and days required to spike emergence were significantly lower than rest of the cultivars, during both the years and in pooled mean basis. The maximum days $(66.00,67.91$ and 66.95) required to spike emergence were observed in cultivar Candyman $\left(\mathrm{V}_{3}\right)$. Interaction between dates of planting and cultivars showed marked difference on spike emergence, during the first year only (Table 2). The minimum (51.00, 54.00 and 52.50) days for spike emergence were noted in 
planting on $30^{\text {th }}$ September with cultivar Dull Queen $\left(D_{1} V_{4}\right)$ during both the years and in pooled mean basis. On the contrary, maximum (68.33) days for spike emergence were noted in $\mathrm{D}_{2} \mathrm{~V}_{3}$ (planting on $15^{\text {th }}$ October with cultivar Candyman) during the first years. And planting on $30^{\text {th }}$ October with cultivar Candyman $\left(\mathrm{D}_{3} \mathrm{~V}_{3}\right)$ showed, maximum (70.00 and 67.50) days for spike emergence during the second years and pooled mean basis. The variation in the response of cultivars on spike emergence may be due to genetic constitution of the cultivars and environmental conditions, during the growing period, viz. higher temperature and long day lengths during end of September than later dates. These findings also collaborate with the results of Arora and Khanna (1985), Arora and Sandhu (1987) and Saleem et al., (2013). Zubair et al., (2006) who reported that different time taken for first spike emergence of the cultivars under prevailing agro-climatic condition and growing of different cultivar with different genetics. Similar findings were also reported by Rao and Janakiram (2006), Swaroop et al., (2005) and Kumar et al., (2008).

\section{Length of spike (cm)}

Planting dates showed significant impact on length of spike, during both the years and on pooled mean basis. The longest length of spike $(72.94,74.36$ and $73.67 \mathrm{~cm})$ was recorded at planting on $30^{\text {th }}$ September $\left(\mathrm{D}_{1}\right)$ during both the years and in pooled mean. However, minimum length of spike $(66.24,68.13$ and $67.20 \mathrm{~cm}$ ) was noted in late planting i.e. on $15^{\text {th }}$ November $\left(D_{4}\right)$ during both the years as well as in pooled mean. Different cultivars of gladiolus differ significantly in producing length of spike. Cultivar Candyman $\left(\mathrm{V}_{3}\right)$ proved significantly superior over rest of the cultivars in producing longest spike length $(84.69,84.68$ and $84.68 \mathrm{~cm})$ during both the years and on pooled mean basis. However, cultivar American Beauty $\left(\mathrm{V}_{1}\right)$ showed the lowest spike length (54.21, 54.06 and 54.15 $\mathrm{cm}$ ), during both the years as well as in pooled mean. The interaction between dates of planting and cultivar on length of spike was found significant during both the years and on pooled mean basis. The longest spike (90.21, 86.73 and $88.47 \mathrm{~cm}$ ) was recorded with the interaction of $30^{\text {th }}$ September planting with cultivar Candyman $\left(D_{1} V_{3}\right)$, during both the years as well as in pooled mean, which was proved significantly superior over rest of the interactions studied in this investigation. However, during second year, interaction $\mathrm{D}_{2} \mathrm{~V}_{5}\left(15^{\text {th }}\right.$ October with cultivar Her Majesty) was found at par with $\mathrm{D}_{1} \mathrm{~V}_{3}$. Shortest spike $(50.03,49.75$ and $49.89 \mathrm{~cm})$ was recorded in planting on $15^{\text {th }}$ November with cultivar American Beauty $\left(\mathrm{D}_{4} \mathrm{~V}_{1}\right)$ during both the years and on pooled mean basis (Table 2). The spike and rachis length of gladiolus is influenced with adequate genetic makeup of the cultivar and availability of adequate nutrient and suitability of environmental factors mainly the light and temperature. Superiority of some genotypes over other genotypes was also reported by Pant and Lal (1991), Arora and Khanna (1985) and Saaie et al., (2011).

\section{Rachis length (cm)}

The longest rachis (52.98, 54.49 and 53.75 $\mathrm{cm})$ was recorded in planting on $30^{\text {th }}$ September $\left(\mathrm{D}_{1}\right)$, during both the years and in pooled mean. The shortest length of rachis $(48.40,49.03$ and $48.71 \mathrm{~cm})$ was observed in planting on $15^{\text {th }}$ November $\left(\mathrm{D}_{4}\right)$, during both the years and on pooled mean. Cultivars also showed significant influence on rachis length. The longest rachis (61.42, 63.92 and 62.67 $\mathrm{cm})$ was obtained in cultivar Candyman $\left(\mathrm{V}_{3}\right)$ which proved its superiority, during both the years and on pooled mean basis. On the contrary, the lowest rachis $(37.35,37.30$, and $37.32 \mathrm{~cm}$ ) was noted in cultivar American Beauty $\left(\mathrm{V}_{1}\right)$, during both the years and in pooled mean. Interaction between dates of 
planting and cultivars was found significant during both the years and on pooled mean basis (Table 2). The longest rachis (66.33, 68.53 and $67.43 \mathrm{~cm}$ ) was obtained in planting on $15^{\text {th }}$ October with cultivar Candyman $\left(\mathrm{D}_{2} \mathrm{~V}_{3}\right)$. However, the shortest rachis (36.00, 35.43 and $35.76 \mathrm{~cm}$ ) was noted in planting on $15^{\text {th }}$ November and cultivar American Beauty $\left(\mathrm{D}_{4} \mathrm{~V}_{1}\right)$ during both the years and on pooled mean. The best quality spike and rachis length were directly correlated with favorable climatic conditions viz. temperature, humidity and photo period during the growth period. The results are in accordance with the findings of Bagde et al., (2009) who reported that planting on $13^{\text {th }}$ October gave batter quality of spike and rachis length.

\section{Number of spike per plant}

Gladiolus planting on $30^{\text {th }}$ September produced the maximum number of spike per plant (1.41, 1.52 and 1.47) and was significantly superior to rest of the dates of planting during both the years as well as on pooled mean basis. However, the minimum number of spike per plant $(1.17,1.21$ and 1.19) was observed in $D_{4}\left(15^{\text {th }}\right.$ November $)$ planting during both the years as well as on pooled mean basis. Gladiolus cultivar American Beauty $\left(\mathrm{V}_{1}\right)$ proved to be the best in producing higher number of spike per plant $(1.85,1.93$ and 1.89) than rest of the cultivars tested, during both the years and on pooled mean basis.

The minimum number of spike (1.05, 1.15 and 1.10) per plant was observed in her Majesty $\left(\mathrm{V}_{5}\right)$ during both the years as well as on pooled mean basis. The interaction between planting dates and cultivars of gladiolus was observed significant during both the years and on pooled mean basis. It was found that planting of $30^{\text {th }}$ September with American Beauty $\left(D_{1} V_{1}\right)$, produced greater number of spike per plant $(2.05,2.09$ and 2.77), and were significantly higher than rest of the treatment combinations of planting dates and cultivars (Table 4) during the second year and on pooled mean basis. The minimum number of spike per plant (1.02) was recorded in planting of $30^{\text {th }}$ October with cultivar Her Majesty $\left(\mathrm{D}_{3} \mathrm{~V}_{5}\right)$, during first years and $15^{\text {th }}$ November with cultivar Her Majesty $\left(\mathrm{D}_{4} \mathrm{~V}_{5}\right)$, was observed minimum number of spike per plant (1.06 and 1.05) during the second year and pooled mean basis. More number of spike per plant produced may be due its genetic constitution of genotypes attributes that might be correlated with growing environment conditions promoted to produce more number of spikes per plant. Simila results was also reported by Kumari et al., (2011).

\section{Days to first floret open}

This attribute was significantly influenced by planting dates during second year. The minimum days required (67.22 and 69.97days) for first floret opening were noted in $30^{\text {th }}$ October planting $\left(\mathrm{D}_{3}\right)$, which was found to be remarkably earlier, during the first year and on pooled mean basis and 71.22 days required with $30^{\text {th }}$ September $\left(D_{1}\right)$, during the second year. On the other hand, maximum days required $(70.27,74.33$ and 72.30 days) to first floret open was observed with planting on $15^{\text {th }}$ November $\left(\mathrm{D}_{4}\right)$ during both years and on pooled mean basis. Gladiolus cultivars exerted significant impact on days to first floret open during both the years and in pooled mean.

Earliest floret opening (62.08, 66.50 and 64.29 days) was observed in cultivar Dull Queen $\left(V_{4}\right)$, which was found significantly earlier than other cultivars during the both year as well as in pooled mean. In case of first year, earlier floret opening was recorded in cultivar Dull Queen $\left(\mathrm{V}_{4}\right)$. On the contrary, the maximum days $(76.75,80.83$ and 78.79) were observed in cultivar Candyman $\left(\mathrm{V}_{3}\right)$, during both the years as well as pooled means. 
Table.1 Effect of dates of planting and cultivars on days to spike emergence, length of spike and rachis length of gladiolus

\begin{tabular}{|c|c|c|c|c|c|c|c|c|c|}
\hline \multirow[t]{2}{*}{ Treatments } & \multicolumn{3}{|c|}{ Days to spike emergence } & \multicolumn{3}{|c|}{ Length of spike (cm) } & \multicolumn{3}{|c|}{ Rachis length (cm) } \\
\hline & 2011-12 & 2012-13 & $\begin{array}{c}\text { Pooled } \\
\text { Mean }\end{array}$ & 2011-12 & 2012-13 & $\begin{array}{c}\text { Pooled } \\
\text { Mean }\end{array}$ & 2011-12 & 2012-13 & $\begin{array}{l}\text { Pooled } \\
\text { Mean }\end{array}$ \\
\hline \multicolumn{10}{|l|}{ Dates of planting } \\
\hline$D_{1}-30$ September & 58.38 & 59.94 & 59.16 & 72.94 & 74.36 & 73.67 & 52.98 & 54.49 & 53.73 \\
\hline $\mathrm{D}_{2}-15$ October & 59.11 & 60.50 & 59.80 & 69.59 & 71.23 & 70.43 & 52.74 & 53.74 & 53.24 \\
\hline $\mathrm{D}_{3}-\mathbf{3 0}$ October & 60.13 & 62.05 & 61.09 & 68.75 & 69.19 & 69.00 & 51.59 & 52.54 & 52.08 \\
\hline$D_{4}-15$ November & 60.33 & 62.55 & 61.45 & 66.24 & 68.13 & 67.20 & 48.40 & 49.03 & 48.71 \\
\hline SEm \pm & 0.42 & 0.56 & 0.35 & 0.56 & 0.44 & 0.36 & 0.49 & 0.47 & 0.33 \\
\hline CD at $5 \%$ & 1.20 & 1.61 & 1.01 & 1.60 & 1.26 & 1.04 & 1.40 & 1.33 & 0.95 \\
\hline \multicolumn{10}{|l|}{ Cultivars } \\
\hline$V_{1}-$ American Beauty & 59.02 & 57.75 & 58.39 & 54.21 & 54.06 & 54.15 & 37.35 & 37.30 & 37.32 \\
\hline V $_{2}-$ White Prosperity & 58.91 & 63.41 & 61.16 & 78.75 & 81.04 & 79.92 & 55.65 & 56.08 & 55.86 \\
\hline $\mathrm{V}_{3}-$ Candyman & 66.00 & 67.91 & 66.95 & 84.69 & 84.68 & 84.68 & 61.42 & 63.92 & 62.67 \\
\hline $\mathbf{V}_{4}-$ Dull Queen & 52.25 & 54.58 & 53.42 & 59.97 & 61.49 & 60.75 & 47.17 & 48.52 & 47.86 \\
\hline $\mathbf{V}_{5}-$ Her Majesty & 57.25 & 58.58 & 57.91 & 64.26 & 67.40 & 65.85 & 47.48 & 48.54 & 48.02 \\
\hline V $_{6}-$ Red Majesty & 63.50 & 65.33 & 64.41 & 74.72 & 75.69 & 75.07 & 59.49 & 60.34 & 59.94 \\
\hline SEm \pm & 0.51 & 0.69 & 0.43 & 0.69 & 0.54 & 0.45 & 0.60 & 0.57 & 0.41 \\
\hline CD at $5 \%$ & 1.46 & 1.97 & 1.23 & 1.96 & 1.55 & 1.28 & 1.72 & 1.63 & 1.17 \\
\hline Interaction D x V & $\mathbf{S}$ & NS & NS & $\mathbf{S}$ & $\mathbf{S}$ & $\mathbf{S}$ & $\mathbf{S}$ & $\mathbf{S}$ & $\mathbf{S}$ \\
\hline
\end{tabular}


Table.2 Interaction effect of dates of planting and cultivars on days to spike emergence, length of spike and Rachis length

\begin{tabular}{|c|c|c|c|c|c|c|c|c|c|}
\hline \multirow{2}{*}{$\begin{array}{l}\text { Treatment } \\
\text { combinations }\end{array}$} & \multicolumn{3}{|c|}{ Days to spike emergence } & \multicolumn{3}{|c|}{ Length of spike (cm) } & \multicolumn{3}{|c|}{ Rachis length (cm) } \\
\hline & 2011-12 & $2012-13$ & Pooled Mean & 2011-12 & $2012-13$ & Pooled Mean & 2011-12 & $2012-13$ & Pooled Mean \\
\hline $\mathrm{D}_{1} \mathrm{~V}_{1}$ & 55.33 & 55.66 & 55.50 & 61.20 & 63.60 & 62.40 & 38.26 & 39.23 & 38.76 \\
\hline$\overline{D_{1} V_{2}}$ & 58.00 & 60.66 & 59.33 & 80.56 & 83.13 & 81.86 & 55.96 & 57.33 & 56.66 \\
\hline$\overline{D_{1} V_{3}}$ & 67.00 & 68.00 & 67.50 & 90.21 & 86.73 & 88.47 & 64.08 & 66.53 & 65.30 \\
\hline$\overline{D_{1} V_{4}}$ & 51.00 & 54.00 & 52.50 & 60.03 & 63.21 & 61.63 & 49.70 & 50.38 & 50.06 \\
\hline $\mathrm{D}_{1} \mathrm{~V}_{5}$ & 55.66 & 57.66 & 56.66 & 66.60 & 70.13 & 68.40 & 50.06 & 52.90 & 51.50 \\
\hline $\mathrm{D}_{1} \mathrm{~V}_{6}$ & 63.33 & 63.66 & 63.50 & 79.06 & 80.10 & 79.63 & 59.83 & 60.60 & 60.23 \\
\hline $\mathrm{D}_{2} \mathrm{~V}_{1}$ & 57.00 & 59.00 & 58.00 & 51.06 & 52.60 & 51.83 & 36.90 & 38.46 & 37.70 \\
\hline $\mathrm{D}_{2} \mathrm{~V}_{2}$ & 58.33 & 63.00 & 60.66 & 80.06 & 81.20 & 80.66 & 55.40 & 57.06 & 56.26 \\
\hline $\mathrm{D}_{2} \mathrm{~V}_{3}$ & 68.33 & 66.00 & 67.16 & 84.34 & 86.00 & 85.17 & 66.33 & 68.53 & 67.43 \\
\hline $\mathrm{D}_{2} \mathrm{~V}_{4}$ & 52.33 & 54.00 & 53.16 & 62.08 & 63.40 & 62.76 & 46.25 & 47.06 & 46.66 \\
\hline$\overline{D_{2} V_{5}}$ & 55.66 & 58.00 & 56.83 & 64.16 & 67.23 & 65.70 & 49.16 & 47.20 & 48.20 \\
\hline $\mathrm{D}_{2} \mathrm{~V}_{6}$ & 63.00 & 63.00 & 63.00 & 75.86 & 76.25 & 76.06 & 62.43 & 64.13 & 63.30 \\
\hline$\overline{\mathrm{D}_{3} \mathrm{~V}_{1}}$ & 61.44 & 56.66 & 59.06 & 54.56 & 50.31 & 52.43 & 38.25 & 36.06 & 37.16 \\
\hline$\overline{D_{3} V_{2}}$ & 58.66 & 64.66 & 61.66 & 80.03 & 81.93 & 80.98 & 55.48 & 55.88 & 55.70 \\
\hline $\mathrm{D}_{3} \mathrm{~V}_{3}$ & 65.00 & 70.00 & 67.50 & 82.16 & 82.46 & 82.33 & 62.20 & 64.78 & 63.53 \\
\hline $\mathrm{D}_{3} \mathrm{~V}_{4}$ & 52.33 & 54.00 & 53.16 & 60.60 & 61.30 & 60.95 & 50.06 & 52.66 & 51.36 \\
\hline $\mathrm{D}_{3} \mathrm{~V}_{5}$ & 58.00 & 60.00 & 59.00 & 62.96 & 67.20 & 65.10 & 45.51 & 47.06 & 46.30 \\
\hline $\mathrm{D}_{3} \mathrm{~V}_{6}$ & 65.33 & 67.00 & 66.16 & 72.20 & 72.50 & 72.36 & 58.02 & 58.78 & 58.43 \\
\hline$\overline{\mathrm{D}_{4} \mathrm{~V}_{1}}$ & 62.33 & 59.66 & 61.00 & 50.03 & 49.75 & 49.89 & 36.00 & 35.43 & 35.76 \\
\hline$\overline{D_{4} V_{2}}$ & 60.66 & 65.33 & 63.00 & 74.33 & 77.91 & 76.13 & 55.76 & 54.06 & 54.96 \\
\hline $\mathrm{D}_{4} \mathrm{~V}_{3}$ & 63.66 & 67.66 & 65.66 & 82.06 & 83.53 & 82.80 & 53.06 & 55.83 & 54.46 \\
\hline $\mathrm{D}_{4} \mathrm{~V}_{4}$ & 53.33 & 56.33 & 54.86 & 57.16 & 58.06 & 57.63 & 42.70 & 44.00 & 43.36 \\
\hline $\mathrm{D}_{4} \mathrm{~V}_{5}$ & 59.66 & 58.66 & 59.16 & 63.33 & 65.06 & 64.20 & 45.18 & 47.00 & 46.10 \\
\hline $\mathrm{D}_{4} \mathrm{~V}_{6}$ & 62.33 & 67.66 & 65.00 & 70.55 & 73.91 & 72.23 & 57.70 & 57.86 & 57.78 \\
\hline SEm \pm & 1.03 & 1.38 & 0.87 & 1.37 & 1.09 & 0.90 & 1.21 & 1.15 & 0.82 \\
\hline $\mathrm{CD}$ at $5 \%$ & 2.93 & NS & NS & 3.90 & 3.11 & 2.56 & 3.45 & 3.27 & 2.33 \\
\hline
\end{tabular}


Table.3 Effect of dates of planting and cultivars on number of spike per plant and Days to first floret open

\begin{tabular}{|c|c|c|c|c|c|c|}
\hline \multirow[t]{2}{*}{ Treatments } & \multicolumn{3}{|c|}{ Number of spike per plant } & \multicolumn{3}{|c|}{ Days to first floret open } \\
\hline & 2011-12 & 2012-13 & $\begin{array}{l}\text { Pooled } \\
\text { Mean }\end{array}$ & 2011-12 & 2012-13 & $\begin{array}{l}\text { Pooled } \\
\text { Mean }\end{array}$ \\
\hline \multicolumn{7}{|l|}{ Dates of planting } \\
\hline $\mathrm{D}_{1}-30$ September & 1.41 & 1.52 & 1.47 & 69.55 & 71.22 & 70.38 \\
\hline $\mathrm{D}_{2}-15$ October & 1.35 & 1.37 & 1.36 & 70.22 & 72.27 & 71.25 \\
\hline $\mathrm{D}_{3}-30$ October & 1.29 & 1.41 & 1.35 & 67.22 & 72.72 & 69.97 \\
\hline$D_{4}-15$ November & 1.17 & 1.21 & 1.19 & 70.27 & 74.33 & 72.30 \\
\hline SEm \pm & 0.02 & 0.02 & 0.02 & 1.77 & 0.46 & 0.93 \\
\hline CD at $5 \%$ & 0.05 & 0.06 & 0.06 & NS & 1.31 & NS \\
\hline \multicolumn{7}{|l|}{ Cultivars } \\
\hline $\mathrm{V}_{1}-$ American Beauty & 1.85 & 1.93 & 1.89 & 69.08 & 68.83 & 68.95 \\
\hline V $_{2}-$ White Prosperity & 1.30 & 1.33 & 1.31 & 65.08 & 74.58 & 69.83 \\
\hline $\mathrm{V}_{3}-$ Candyman & 1.27 & 1.34 & 1.30 & 76.75 & 80.83 & 78.79 \\
\hline $\mathbf{V}_{4}-$ Dull Queen & 1.22 & 1.32 & 1.27 & 62.08 & 66.50 & 64.29 \\
\hline $\mathrm{V}_{5}-$ Her Majesty & 1.05 & 1.15 & 1.10 & 68.08 & 69.25 & 68.66 \\
\hline V $_{6}-$ Red Majesty & 1.14 & 1.22 & 1.18 & 74.83 & 75.83 & 75.33 \\
\hline SEm \pm & 0.03 & 0.02 & 0.02 & 2.17 & 0.56 & 1.14 \\
\hline CD at $5 \%$ & 0.08 & 0.05 & 0.05 & 6.18 & 1.60 & 3.25 \\
\hline Interaction D x V & $\mathbf{S}$ & $\mathbf{S}$ & $\mathbf{S}$ & NS & NS & NS \\
\hline
\end{tabular}


Table.4 Interaction effect of dates of planting and cultivars on number of spike per plant and Days to first floret open

\begin{tabular}{|c|c|c|c|c|c|c|}
\hline \multirow{2}{*}{$\begin{array}{l}\text { Treatment } \\
\text { combinations }\end{array}$} & \multicolumn{3}{|c|}{ Number of spike per plant } & \multicolumn{3}{|c|}{ Days to first floret open } \\
\hline & 2011-12 & 2012-13 & Pooled Mean & 2011-12 & 2012-13 & Pooled Mean \\
\hline$\overline{D_{1} V_{1}}$ & 2.05 & 2.09 & 2.07 & 67.33 & 67.66 & 67.50 \\
\hline $\mathrm{D}_{1} \mathrm{~V}_{2}$ & 1.40 & 1.42 & 1.41 & 70.33 & 72.00 & 71.16 \\
\hline $\mathrm{D}_{1} \mathrm{~V}_{3}$ & 1.42 & 1.55 & 1.48 & 78.33 & 81.00 & 79.66 \\
\hline $\mathbf{D}_{1} \mathbf{V}_{4}$ & 1.38 & 1.46 & 1.42 & 60.33 & 64.66 & 62.50 \\
\hline $\mathrm{D}_{1} \mathrm{~V}_{5}$ & 1.08 & 1.28 & 1.18 & 65.66 & 67.66 & 66.66 \\
\hline $\mathrm{D}_{1} \mathrm{~V}_{6}$ & 1.18 & 1.31 & 1.24 & 75.33 & 74.33 & 74.83 \\
\hline$\overline{D_{2} V_{1}}$ & 1.96 & 2.01 & 1.98 & 69.00 & 70.33 & 69.66 \\
\hline $\mathrm{D}_{2} \mathrm{~V}_{2}$ & 1.35 & 1.36 & 1.35 & 70.33 & 75.00 & 72.66 \\
\hline $\mathrm{D}_{2} \mathrm{~V}_{3}$ & 1.35 & 1.24 & 1.29 & 77.66 & 78.66 & 78.16 \\
\hline $\mathrm{D}_{2} \mathrm{~V}_{4}$ & 1.20 & 1.33 & 1.26 & 62.33 & 66.00 & 64.16 \\
\hline $\mathrm{D}_{2} \mathrm{~V}_{5}$ & 1.40 & 1.13 & 1.08 & 67.33 & 69.33 & 68.33 \\
\hline $\mathrm{D}_{2} \mathrm{~V}_{6}$ & 1.15 & 1.15 & 1.15 & 74.66 & 74.33 & 74.50 \\
\hline $\mathrm{D}_{3} \mathrm{~V}_{1}$ & 1.93 & 2.03 & 1.99 & 70.33 & 66.66 & 68.50 \\
\hline $\mathrm{D}_{3} \mathrm{~V}_{2}$ & 1.26 & 1.40 & 1.33 & 49.00 & 75.66 & 62.33 \\
\hline $\mathrm{D}_{3} \mathrm{~V}_{3}$ & 1.20 & 1.33 & 1.26 & 76.33 & 81.66 & 79.00 \\
\hline $\mathrm{D}_{3} \mathrm{~V}_{4}$ & 1.11 & 1.28 & 1.23 & 62.66 & 65.00 & 63.83 \\
\hline $\mathrm{D}_{3} \mathrm{~V}_{5}$ & 1.02 & 1.13 & 1.17 & 69.33 & 70.66 & 70.00 \\
\hline $\mathrm{D}_{3} \mathrm{~V}_{6}$ & 1.13 & 1.28 & 1.20 & 75.66 & 76.66 & 76.16 \\
\hline $\mathrm{D}_{4} \mathbf{V}_{1}$ & 1.48 & 1.55 & 1.51 & 69.66 & 70.66 & 70.16 \\
\hline $\mathrm{D}_{4} \mathrm{~V}_{2}$ & 1.15 & 1.13 & 1.14 & 70.66 & 75.66 & 73.16 \\
\hline $\mathbf{D}_{4} \mathbf{V}_{3}$ & 1.11 & 1.20 & 1.15 & 74.66 & 82.00 & 78.33 \\
\hline $\mathrm{D}_{4} \mathbf{V}_{4}$ & 1.11 & 1.22 & 1.16 & 63.00 & 70.33 & 66.66 \\
\hline $\mathrm{D}_{4} \mathrm{~V}_{5}$ & 1.04 & 1.06 & 1.05 & 70.00 & 69.33 & 69.66 \\
\hline $\mathrm{D}_{4} \mathrm{~V}_{6}$ & 1.08 & 1.13 & 1.10 & 73.66 & 78.00 & 75.83 \\
\hline SEm \pm & 0.06 & 0.05 & 0.05 & 4.35 & 1.12 & 2.29 \\
\hline CD at $5 \%$ & 017 & 0.14 & 0.14 & NS & NS & NS \\
\hline
\end{tabular}


Table.5 Effect of dates of planting and cultivars on of florets per spike, diameter of florets and internodal length of florets of gladiolus

\begin{tabular}{|c|c|c|c|c|c|c|c|c|c|}
\hline \multirow[t]{2}{*}{ Treatments } & \multicolumn{3}{|c|}{ No. of florets per spike } & \multicolumn{3}{|c|}{ Diameter of florets(cm) } & \multicolumn{3}{|c|}{ Internodal length (cm) } \\
\hline & 2011-12 & 2012-13 & $\begin{array}{c}\text { Pooled } \\
\text { Mean }\end{array}$ & 2011-12 & 2012-13 & $\begin{array}{c}\text { Pooled } \\
\text { Mean }\end{array}$ & 2011-12 & 2012-13 & $\begin{array}{l}\text { Pooled } \\
\text { Mean }\end{array}$ \\
\hline \multicolumn{10}{|l|}{ Dates of Planting } \\
\hline$D_{1}-30$ September & 13.44 & 14.17 & 13.81 & 10.51 & 10.59 & 10.55 & 4.60 & 3.99 & 4.30 \\
\hline$\overline{D_{2}-15 \text { October }}$ & 13.23 & 13.64 & 13.44 & 10.49 & 9.64 & 10.07 & 4.53 & 3.96 & 4.26 \\
\hline $\mathrm{D}_{3^{-}} 30$ October & 12.55 & 12.97 & 12.78 & 10.48 & 9.51 & 10.00 & 4.66 & 3.87 & 4.26 \\
\hline$D_{4}$ - 15 November & 12.03 & 12.63 & 12.34 & 10.01 & 9.49 & 9.75 & 4.52 & 3.80 & 4.16 \\
\hline SEm \pm & 0.18 & 0.14 & 0.10 & 0.12 & 0.09 & 0.08 & 0.07 & 0.04 & 0.04 \\
\hline CD & 0.52 & 0.40 & 0.28 & 0.35 & 0.27 & 0.24 & NS & 0.13 & NS \\
\hline \multicolumn{10}{|l|}{ Cultivars } \\
\hline $\mathrm{V}_{1}-$ American beauty & 10.87 & 11.58 & 11.23 & 9.43 & 9.29 & 9.35 & 4.33 & 3.76 & 4.04 \\
\hline $\mathrm{V}_{2^{-}}$white prosperity & 14.47 & 14.66 & 14.57 & 10.83 & 9.95 & 10.38 & 4.43 & 4.03 & 4.24 \\
\hline $\mathrm{V}_{3}-$ Candy man & 14.85 & 15.17 & 15.02 & 10.92 & 10.43 & 10.67 & 5.04 & 4.37 & 4.70 \\
\hline V $_{\text {4-Dull Queen }}$ & 11.85 & 12.72 & 12.30 & 10.14 & 9.34 & 9.75 & 4.28 & 3.32 & 3.80 \\
\hline $\mathrm{V}_{5^{-}}$Her majesty & 12.12 & 12.74 & 12.44 & 10.20 & 9.23 & 9.72 & 4.56 & 3.66 & 4.13 \\
\hline $\mathrm{V}_{6^{-}}$Red majesty & 12.73 & 13.25 & 13.00 & 10.92 & 10.60 & 10.76 & 4.83 & 4.30 & 4.56 \\
\hline SEm \pm & 0.22 & 0.17 & 0.12 & 0.15 & 0.11 & 0.10 & 0.08 & 0.06 & 0.05 \\
\hline CD at $5 \%$ & 0.63 & 0.50 & 0.35 & 0.43 & 0.33 & 0.29 & 0.24 & 0.17 & 0.15 \\
\hline Interaction D x V & $\mathbf{S}$ & $\mathbf{S}$ & $\mathbf{S}$ & $\mathbf{S}$ & $\mathbf{S}$ & $\mathbf{S}$ & NS & $\mathbf{S}$ & $\mathbf{S}$ \\
\hline
\end{tabular}


Table.6 Interaction effect of dates of planting and cultivars on of florets per spike, diameter of florets and internodal length of florets of gladiolus

\begin{tabular}{|c|c|c|c|c|c|c|c|c|c|}
\hline \multirow{2}{*}{$\begin{array}{l}\text { Treatment } \\
\text { combinations }\end{array}$} & \multicolumn{3}{|c|}{ No. of florets per spike } & \multicolumn{3}{|c|}{ Diameter of florets(cm) } & \multicolumn{3}{|c|}{ Internodal length (cm) } \\
\hline & 2011-12 & 2012-13 & Pooled Mean & 2011-12 & 2012-13 & Pooled Mean & 2011-12 & 2012-13 & Pooled Mean \\
\hline $\mathbf{D}_{1} \mathbf{V}_{1}$ & 11.55 & 11.86 & 11.70 & 9.24 & 9.86 & 9.53 & 4.61 & 3.62 & 4.13 \\
\hline $\mathrm{D}_{1} \mathrm{~V}_{2}$ & 14.80 & 15.33 & 15.06 & 10.60 & 11.31 & 10.93 & 4.32 & 3.90 & 4.13 \\
\hline $\mathrm{D}_{1} \mathbf{V}_{3}$ & 14.96 & 15.86 & 15.43 & 11.14 & 11.49 & 11.33 & 5.27 & 4.74 & 5.00 \\
\hline $\mathrm{D}_{1} \mathbf{V}_{4}$ & 13.61 & 14.20 & 13.93 & 9.98 & 10.07 & 10.03 & 4.50 & 3.46 & 3.96 \\
\hline $\mathrm{D}_{1} \mathrm{~V}_{5}$ & 12.05 & 13.71 & 12.90 & 10.30 & 9.29 & 9.80 & 4.30 & 3.89 & 4.10 \\
\hline $\mathrm{D}_{1} \mathrm{~V}_{6}$ & 13.66 & 14.06 & 13.86 & 11.88 & 11.54 & 11.70 & 4.63 & 4.34 & 4.48 \\
\hline $\mathrm{D}_{2} \mathrm{~V}_{\mathbf{1}}$ & 10.73 & 11.46 & 11.10 & 9.54 & 9.35 & 9.43 & 4.07 & 3.92 & 4.03 \\
\hline $\mathrm{D}_{2} \mathrm{~V}_{2}$ & 14.52 & 14.60 & 14.56 & 11.03 & 9.33 & 10.16 & 4.34 & 4.18 & 4.23 \\
\hline $\mathrm{D}_{2} \mathrm{~V}_{3}$ & 15.40 & 15.58 & 15.50 & 11.17 & 10.74 & 10.96 & 4.76 & 4.13 & 4.46 \\
\hline $\mathrm{D}_{2} \mathrm{~V}_{4}$ & 12.06 & 13.25 & 12.66 & 10.03 & 8.99 & 9.53 & 4.42 & 3.44 & 3.93 \\
\hline $\mathrm{D}_{2} \mathrm{~V}_{5}$ & 12.43 & 12.60 & 12.53 & 10.01 & 9.08 & 9.53 & 4.79 & 3.93 & 4.40 \\
\hline $\mathrm{D}_{2} \mathrm{~V}_{6}$ & 14.26 & 14.35 & 14.30 & 11.18 & 10.37 & 10.80 & 4.82 & 4.17 & 4.50 \\
\hline $\mathrm{D}_{\mathbf{3}} \mathrm{V}_{\mathbf{1}}$ & 10.98 & 11.40 & 11.19 & 9.74 & 9.43 & 9.58 & 4.28 & 3.58 & 3.93 \\
\hline $\mathrm{D}_{3} \mathrm{~V}_{2}$ & 14.43 & 14.76 & 14.60 & 11.10 & 9.89 & 10.50 & 4.42 & 3.86 & 4.16 \\
\hline $\mathrm{D}_{\mathbf{3}} \mathbf{V}_{\mathbf{3}}$ & 14.66 & 14.78 & 14.73 & 11.25 & 10.42 & 10.80 & 5.16 & 4.35 & 4.76 \\
\hline $\mathrm{D}_{3} \mathrm{~V}_{4}$ & 10.66 & 12.10 & 11.40 & 9.86 & 8.91 & 9.36 & 4.38 & 3.26 & 3.83 \\
\hline $\mathrm{D}_{3} \mathrm{~V}_{5}$ & 11.93 & 12.00 & 11.96 & 10.80 & 9.18 & 10.03 & 4.62 & 3.33 & 4.00 \\
\hline $\mathrm{D}_{3} \mathrm{~V}_{6}$ & 12.66 & 13.20 & 12.96 & 10.17 & 10.12 & 10.16 & 5.10 & 4.46 & 4.80 \\
\hline $\mathrm{D}_{4} \mathrm{~V}_{1}$ & 10.22 & 11.00 & 10.61 & 9.19 & 8.53 & 8.86 & 4.16 & 3.93 & 4.03 \\
\hline $\mathrm{D}_{4} \mathrm{~V}_{2}$ & 14.13 & 13.96 & 14.06 & 10.58 & 9.28 & 9.93 & 4.66 & 4.18 & 4.43 \\
\hline $\mathrm{D}_{4} \mathbf{V}_{\mathbf{3}}$ & 14.38 & 14.45 & 14.43 & 10.13 & 9.10 & 9.60 & 4.96 & 4.40 & 4.66 \\
\hline $\mathrm{D}_{4} \mathrm{~V}_{4}$ & 11.06 & 11.36 & 11.23 & 10.71 & 9.40 & 10.06 & 4.03 & 2.50 & 3.26 \\
\hline $\mathrm{D}_{4} \mathrm{~V}_{5}$ & 12.06 & 12.65 & 12.36 & 9.70 & 9.37 & 9.53 & 4.53 & 2.50 & 3.51 \\
\hline $\mathrm{D}_{4} \mathrm{~V}_{6}$ & 10.33 & 12.00 & 11.16 & 9.70 & 10.36 & 10.03 & 4.76 & 3.12 & 3.82 \\
\hline SEm \pm & 0.44 & 0.35 & 0.24 & 0.30 & 0.23 & 0.20 & 0.17 & 0.12 & 0.11 \\
\hline CD at $5 \%$ & 1.26 & 1.00 & 0.69 & 0.86 & 0.67 & 0.58 & NS & 0.34 & 0.31 \\
\hline
\end{tabular}


Interaction effect between dates of planting and cultivars to first floret open was showed non-significant, during both the years and in pooled mean. Variation in first floret open might be attributed to difference in genetic constituent of genotypes and it might also be influenced by their interaction with growing environmental conditions. The present findings are in the conformity with the findings of Arora and Sandhu (1987). Similar results were obtained by Kem et al., (2003), Swaroop et al., (2005) and Zubair et al., (2006).

\section{Number of floret per spike}

Significant difference in number of florets per spike was observed with different date of planting. The maximum number of florets per spike (13.44, 14.17 and 13.81) was exhibited under planting on $30^{\text {th }}$ September $\left(D_{1}\right)$, during both the years and on pooled mean basis. The minimum numbers of floret per spike (12.03, 12.63 and 12.34) were noted in planting on $15^{\text {th }}$ November $\left(D_{4}\right)$. Different cultivars of gladiolus exhibited significant impact in respect to number of florets per spike. The maximum numbers of floret per spike (14.85, 15.17 and 15.02) were observed in cultivar Candyman $\left(\mathrm{V}_{3}\right)$, during both the years and as on pooled mean basis. However, minimum number of florets per spike $(10.87,11.58$ and 11.23) was noted in cultivar American Beauty $\left(\mathrm{V}_{1}\right)$. Interaction effect of the different dates of planting and cultivars in respect to number of florets per spike was found significant, during both the years as well as on pooled basis (Table 4). The maximum number of floret per spike (15.40 and 15.50) was recorded in planting on $15^{\text {th }}$ October with cultivar Candyman $\left(\mathrm{D}_{2} \mathrm{~V}_{3}\right)$, during first year and on pooled mean basis. However, during second year highest number of florets per spike (15.86) was obtained in planting on $30^{\text {th }}$ September with cultivar Candyman $\left(D_{1} V_{3}\right)$. On the contrary, the minimum number of floret (10.22, 11.00 and 10.61) was noted in planting on $15^{\text {th }}$ November with cultivar American beauty $\left(\mathrm{D}_{4} \mathrm{~V}_{1}\right)$. The variation in number of florets per spike might be due to hereditary traits of the genotypes and it is directly correlated with favorable climatic conditions viz. temperature, humidity and photo period during the growth period. The results are in accordance with the findings of Rani and Singh (2005), Bagde et al., (2009), Kumari et al., (2011) and saaie et al., (2011).

\section{Diameter of floret $(\mathrm{cm})$}

Significantly the maximum diameter of floret $(10.51,10.59$ and $10.55 \mathrm{~cm})$ was recorded in planting on $30^{\text {th }}$ September $\left(D_{1}\right)$ which was significantly higher than the rest of planting dates, during both the years and on pooled mean basis. The minimum diameter of floret $(10.01,9.49$ and $9.75 \mathrm{~cm})$ was noted in planting on $15^{\text {th }}$ November $\left(\mathrm{D}_{4}\right)$ during both the years and in pooled mean basis. The maximum diameter of floret (10.92, 10.60 and $10.76 \mathrm{~cm}$ ) was noted in cultivar Red Majesty $\left(\mathrm{V}_{6}\right)$ and was found at par with cultivar Candyman $\left(\mathrm{V}_{3}\right)$, during both the years and on pooled mean basis. The minimum diameter of floret $(9.43,9.29$ and 9.35) was observed in cultivar American Beauty $\left(\mathrm{V}_{1}\right)$, during the both year as well as on pooled mean basis. Interaction between different dates of planting and cultivars were observed significant during both the years and on pooled mean. Significantly maximum values $(11.88,11.54$ and 11.70) were recorded in planting of $30^{\text {th }}$ September with cultivar Red Majesty $\left(D_{1} V_{6}\right)$, which was significantly higher than rest of treatment combinations except $\mathrm{D}_{1} \mathrm{~V}_{3}, \mathrm{D}_{2} \mathrm{~V}_{3}$, $\mathrm{D}_{2} \mathrm{~V}_{6}, \mathrm{D}_{3} \mathrm{~V}_{2}$ and $\mathrm{D}_{3} \mathrm{~V}_{3}$ during the first year, $D_{1} V_{2}, D_{1} V_{3}$ and $D_{2} V_{3}$ in the second year and $\mathrm{D}_{1} \mathrm{~V}_{3}$ on pooled mean basis. The minimum diameter of floret $(9.19,8.53$ and 8.86$)$ was noted in planting of $15^{\text {th }}$ November with cultivar American Beauty $\left(\mathrm{D}_{4} \mathrm{~V}_{1}\right)$ during the both year as well as on pooled mean basis. 
The variation in floret diameter might be due to attributed to difference in genetics constituent of different genotypes and it might also be influenced by their interaction with growing environmental conditions. The results also support from findings of Baweja and Brahma (2003) and Kumar and Yadav (2005) and Zubair et al., (2006).

\section{Internodal length}

Internodal length was not influenced by dates of planting during the first year and in pooled mean basis. However, it was influenced significantly due to dates of planting during the second year. Planting on $15^{\text {th }}$ November $\left(\mathrm{D}_{4}\right)$ showed shortest $(4.52,3.80$ and $4.16 \mathrm{~cm})$ during the both year as well as on pooled mean basis. And the maximum internodal length $(4.66 \mathrm{~cm})$ was recorded with $30^{\text {th }}$ October $\left(\mathrm{D}_{3}\right)$, during the first year. However the maximum internodal length (3.99 and $4.30)$ were recorded with $30^{\text {th }}$ September $\left(D_{1}\right)$, during the second year and pooled mean basis. Different cultivars showed significant impact on intermodal length during both the years and in pooled mean. The maximum internodal length $(5.04,4.37$ and $4.70 \mathrm{~cm})$ was recorded in cultivar Candyman $\left(\mathrm{V}_{3}\right)$. The minimum intermodal length $(4.28,3.32$ and $3.80 \mathrm{~cm}$ ) was observed in cultivar Dull Queen $\left(\mathrm{V}_{4}\right)$, which was significantly inferior to rest of the cultivars. The interaction between dates of planting and cultivars on internodal length was found non-significant difference, during the first year. Although this character was influenced significantly by the interaction during the second year and on pooled means (Table 6). The longest internodal length (4.74 and $5.00 \mathrm{~cm}$ ) was noted under planting on $30^{\text {th }}$ September with cultivar Candyman $\left(D_{1} V_{3}\right)$ during, second year as well as in pooled mean. The minimum length of internodes $(4.03,2.50$ and $3.26 \mathrm{~cm})$ was recorded under planting on $15^{\text {th }}$ November with cultivar Dull Queen $\left(D_{4} V_{4}\right)$. These flower characters might be due to inheritance of genotypic character of the cultivar. It is also depends on growing environment condition, hence cultivars of gladiolus which perform well in one region may not perform same in other regions of varying climatic conditions. Similar finding were also reported by Kumar and Yadav (2005) and Saleem et al., (2013).

\section{References}

Arora, J.S. and Khanna, K. 1985. Evaluation of gladiolus cultivars. J. Res. Punjab Agri. Univ. 22 (4): 655-62.

Arora, J.S. and Sandhu, G.S. 1987. Effect of two planting dates on the performance of fifteen gladiolus cultivars. The Punjab Hort. Journal, 27 (4): 243-249.

Bagde, M.S., Golliwar, V.J., Yadgirwar, B.M. and Wankhede, M.N. 2009. Effect of planting dates on flower quality and yield parameters of gladiolus. J. soils and crops, 19 (2): 351-354.

Baweja, H.S. and Brahma, B. 2003. Performance of some gladiolus cultivar under mid hill conditions of Himachal Pradesh. Scientia Horticulture, 8: 191197.

Kem, J.C., Yadav, S. K., and Kumar, S. 2003. Performance of gladiolus cultivars under Valley conditions of Uttaranchal. Progressive Hort. Vol. 35 (1) 108 - 110.

Kumar, P; Kumar, R. and Kumar, A. 2008. Effect of organic culture on growth, development and post-harvest life of gladiolus (Gladiolus hybrid). J. Ornam. Hort. 11 (2):127-130.

Kumar, R. and Yadav, D.S. 2005, Evaluation of gladiolus cultivars under subtropical hills of Meghalaya. J. Ornam. Hort., 8(2): 86-90.

Kumari, S. and Patel, B.S. and Mahawer, L.N. 2011. Influence of gebberallic acid and planting dates on vegetative growth and flower production in gladiolus cv. 
Yellow Frilled. Prog Hort. 43 (2): 219224.

Lepcha, B., Naufiyal, M.E. and Rao, V.K. 2007. Variability studies in gladiolus under mid hill conditions of Uttarakhand. J. Ornam. Hort.10 (3): $169-172$

Pant, C.C. and Lal, S.D. 1991. Genetic Variability in Gladiolus. Prog. Hort. 23: $1-4$.

Rani R, Singh C, Evaluation of different gladiolus cultivars for quality flower production2005. J. Res. Birsa Agric Univ, 17(2), p 227-230.

Rao, T. M. and Janakiram, T. 2006. Performance of exotic Orchidiolus and I.I.H.R. gladiolus Cultivars J. Ornam. Horti., 9(1):61-62.

Saaie, M.S., Ahlawat, V.P., Sehrawat, S.K., Sindhu, S.S. and Yadav, B.S. 2011. Studies on cultivation of gladiolus in open and protected conditions. Haryana J. hortic. Sci., 40 (3\&4): 160-163.

Saleem, M., Ahmad, I. and Khan, M.A. 2013. Cultivar effect on growth, yield and cormel production of gladiolus (Gladiolus grandiflorus L.) J. Ornam. \& Hort. Plants, 3(1): 39-48.

Sharma, S.C. and Sharga, A.N. 1994. Floriculture-Technology, Trades and Trends, Oxford and ABH Pub., New Delhi.

Swaroop, K., Krishana, Singh P. and Singh, K. 2005. Performance of gladiolus under Delhi conditions. J. Ornam. Hort. 8(1): 32-35.

Usha Bala, chandna sekhar, T.R and Reddy. Y.N. 2002, Vase life studies of three gladiolus cultivars as influenced by dates of planting and iron sulphate spray. Journal of Research ANGRAU, 30 (3): 40-43.

Zubair, M., Wazir, F., Khan, Akhatar Sohail and Ayub Gohar, 2006. Planting dates affect floral characteristics of gladiolus under the Soil and climatic condition of Peshawar. Pakistan J. Bio. Sciences 9 (9): 1669-1676.

\section{How to cite this article:}

Tirkey T., Sameer Tamrakar, Gaurav Sharma and Mukesh Sahu. 2018. Effect of Planting Dates and Cultivars on Floral Characters of Gladiolus (Gladiolus grandiflorus) under Chhattisgarh Plains. Int.J.Curr.Microbiol.App.Sci. 7(06): 1964-1976.

doi: https://doi.org/10.20546/ijcmas.2018.706.233 